\title{
Kinetic approach to one-dimensional non-uniform granular gases
}

\author{
Duanming Zhang ${ }^{1}$, Rui Li ${ }^{1}$, Xiangying Su${ }^{1}$, Guijun $\operatorname{Pan}^{1}$ and \\ Boming $\mathbf{Y u}^{1,2}$ \\ ${ }^{1}$ Department of Physics, Huazhong University of Science and Technology, 1037 Luoyu Road, \\ Wuhan 430074, People's Republic of China \\ 2 The State Key Laboratory of Nonlinear Mechanics (LNM), Institute of Mechanics, \\ Chinese Academy of Science, Beijing 100080, People's Republic of China \\ E-mail: liruilr@263.net
}

Received 29 March 2005, in final form 30 August 2005

Published 28 September 2005

Online at stacks.iop.org/JPhysA/38/8861

\begin{abstract}
We present a model of polydisperse granular mixture with a power-law size distribution subjected to Brownian forces. The driven inelastic mixture which has $n$ components can be regarded as 'granular gas'. The stationary state where the mixture reaches is the result of the balance between the dissipation and random forces which inject energies. We have studied the nonequilibrium properties of the system by means of Monte Carlo method. When the typical relaxation time $\tau$ of the driving Brownian process is greater than the mean collision time $\tau_{c}$, the velocity probability distribution strongly deviates from the Gaussian one and the system has a strong spatial clustering. We define the partial granular temperature and the global granular temperature of the multi-component mixture. The partial granular temperature is the average kinetic energy per particle of the same component and the global one is the statistic average value per partial granular temperature of the mixture. One of the most useful properties of the temperature, i.e., the independence from the thermal substance, is lost. We introduce a fractal dimension $D$ as a measurement of the inhomogeneity of the size distribution of the mixture. Our simulations represent that the deviation and clusterization become more and more pronounced with the increasing value of the fractal dimension $D$. The global granular temperature decreases, and dissipated energy per particle increases as $D$ augments.
\end{abstract}

PACS numbers: 05.45.Df, 61.43.Bn, 61.43.Hv 


\section{Introduction}

Granular systems are widespread in nature and a fundamental understanding of the dynamics of granular materials still poses a challenge for physicists and engineers [1-4]. The key feature that distinguishes granular systems from conventional solids, liquids and gases is that the interactions between grains dissipate energy [3]. The energy loss is at the base of many interesting phenomena, such as inelastic collapse [6], deviation from Maxwellian behaviour (observed experimentally in different geometrics and for different driving conditions in [7-11] and in numerical simulations [13-17]) and clustering (for a sample of theoretical, simulational and experimental approaches see [8, 9, 12, 18-20]). Du, Li and Kandanoff [18] who considered $N$ identical hard rods confined between a thermal and a reflecting wall. In the model one has a statistically steady state as a result of the balance between the dissipation of the kinetic energy due to the collisions between the rods and the energy reinjection due to the thermal wall; the latter supplies energy only to the last particle, which in turn transfers energy and momentum to the rest of the system, producing a trivial cluster near the opposite wall. Such a state represents a breakdown of the equipartition of the energy in a stationary nonequibrium system. In this model, the mean kinetic energy per particle,

$$
E=\frac{1}{2 N} \sum_{i=1}^{N}\left\langle v_{i}(t)^{2}\right\rangle,
$$

and the mean dissipated energy per particle per unit time,

$$
W=\frac{1}{\Delta t} \sum_{j}\left\langle(\Delta E)_{j}\right\rangle
$$

(where $(\Delta E)_{j}$ is the energy loss during the $j$ th collision occurred in the time interval $[t-\Delta t / 2, t+\Delta t / 2]$, and \langle\rangle is the time average), are not independent of the total number of particles, but decay exponentially with the number $N$ of particles.

Williams and MacKintosh [23] proposed an alternative heating mechanism. The idea is to supply kinetic energy to every particle by means of a random acceleration at every time step. Though this method is numerically efficient, it does not appear realistic from a physical point of view. In the spirit of the one introduced by Du, Li and Kandanoff, Puglisi et al [21, 22] presented a model of driven granular gases subjected to Brownian forces to investigate the clustering and non-Gaussian behaviour of the granular gases. The stationary state for this model is the result of the balance between the dissipation and the random forces which inject energies. It has two regimes [21, 22]: when the typical relaxation time $\tau$ of the driving Brownian process is smaller than the mean collision time $\tau_{c}$, the spatial density is nearly homogeneous and the velocity probability distribution is Gaussian. In the opposite limit, i.e., $\tau \gg \tau_{c}$, it has a strong spatial clustering and the velocity probability distribution strongly deviates from the Gaussian distribution. These phenomena are more and more pronounced with decreasing values of the restitution coefficient $e$. This model is only applied to a uniform granular system. However, in actual granular systems the sizes of particles are different. Some authors [5, 24, 25] discussed two-component granular mixtures and found the appearance of two different granular temperatures, one for each species. Zhang et al [26-28] presented a fractal model of non-uniform granular system. They discussed that the fractal dimension $D$ influences the carrying characteristics and dynamic behaviours of particles because of different size distributions.

In this paper, we present a model of polydisperse-driven inelastic granular mixture with a power-law size distribution subjected to Brownian forces. We apply the Monte Carlo method in the case far from the equilibrium to discuss the effects on the dynamic behaviours of particles 
Table 1. Relationship between the granular size dispersion and the fractal dimension $D$.

\begin{tabular}{lccccccc}
\hline & \multicolumn{7}{c}{ Size distribution $^{\mathrm{b}}(\%)$} \\
\cline { 2 - 7 } Mud formulation $^{\mathrm{a}}$ & $<3.3 \mu \mathrm{m}$ & $<4.7 \mu \mathrm{m}$ & $<9.4 \mu \mathrm{m}$ & $<13.0 \mu \mathrm{m}$ & $<19.0 \mu \mathrm{m}$ & $<27.0 \mu \mathrm{m}$ & $D$ \\
\hline $2 \%$ bentonite & 24.9 & 39.4 & 62.3 & 72.0 & 77.6 & 100.0 & 2.39 \\
$4 \%$ bentonite & 32.0 & 50.6 & 73.5 & 83.9 & 88.4 & 100.0 & 2.50 \\
$6 \%$ bentonite & 42.2 & 66.6 & 80.8 & 86.9 & 88.4 & 100.0 & 2.65 \\
$6.5 \%$ bentonite $+2 \% \mathrm{NaCl}$ & 38.4 & 60.6 & 79.1 & 85.9 & 90.8 & 100.0 & 2.59 \\
$6.5 \%$ bentonite $+4 \% \mathrm{NaCl}$ & 31.9 & 50.4 & 72.4 & 82.8 & 88.4 & 100.0 & 2.49 \\
$6.5 \%$ bentonite $+6 \% \mathrm{NaCl}$ & 25.4 & 40.9 & 68.7 & 82.9 & 89.1 & 100.0 & 2.36 \\
\hline
\end{tabular}

${ }^{a}$ The muds were aged for $24 \mathrm{~h}$ at room temperature prior to use.

${ }^{\mathrm{b}}$ Determined by using a microtrac laser particle size analyser.

induced by the fractal characteristics. We investigate how the dynamic behaviour of the driven granular gas changes with the inhomogeneity of size distribution. Furthermore, we define the granular temperature of the multi-mixture and discuss how the granular temperature and the average dissipated energy per particle change with the inhomogeneity of the system.

\section{One-dimensional model of non-uniform driven inelastic granular system with fractal characteristic}

We consider a polydisperse granular mixture with a power-law size distribution. We assume the mass distribution of $N$ particles is continuous $\left(m_{\min } \leqslant m \leqslant m_{\max }\right.$, where $m_{\min }$ is the minimal mass of the particles, and $m_{\max }$ is the maximal mass of the particles). $N_{i}$ is the number of particles of the $i$ th component, and $N_{1}+N_{2}+\cdots+N_{i}+\cdots=N$. The mass of particles of the $i$ th component is $m_{i}$. For simplicity, the surface of the hard particles is smooth. The material of each particle is identical, but the size of particles is different. The mass of a particle is given by

$$
m=\frac{4}{3} \pi r^{3} \rho_{p},
$$

where $\rho_{p}$ and $r$ are the mass density and the radius of the particles, respectively.

Experiments show that the granular materials exhibit some fractal characteristics. When $n_{0} / N \ll 1, n_{0}$ is the number of particles with the maximum size $r_{\max }$; the size distribution of particles in granular system satisfies the size-frequency character by [26]:

$$
Y_{n_{r}}(r)=1-N^{-1} n_{0}\left(\frac{r}{r_{\max }}\right)^{-D},
$$

where $Y_{n_{r}}$ is the ratio of $n_{r}$ to $N, n_{r}$ is the number of particles whose size is smaller than $r, N$ is the total number of particles and $D$ is the fractal dimension, $2<D<3$. From equations (3) and (4), the mass of any particle in the mixture can be given by

$$
m=m_{\max }\left[\frac{N}{n_{0}}\left(1-Y_{N_{r}}\right)\right]^{-3 / D} .
$$

If the values of $N, m_{\max }, n_{0}$ and $D$ are given, according to equation (5), we can randomly evaluate the mass per particle in the non-uniform granular system with fractal distribution.

In [30] Zhang et al discussed the relationship between the granular size dispersion of the non-uniform granular system and the fractal dimension $D$ of the size distribution according to the experimental granulometric analysis. Table 1 lists the size distribution [30]. 
From the above data shown in table 1 , it can be seen that, both in a simplex non-uniform granular system and in a mixed granular system, the higher $D$ implies the greater dispersion of the finer particles, which leads to more inhomogeneity in the size distribution due to the formation of relatively fine particles. That is to say, the fractal dimension $D$ can be considered as a measurement of the inhomogeneity of the size distribution. The granular system will be more and more inhomogenous with the increasing value of the fractal dimension $D$.

To study the actual granular gas with fractal characteristic, we present the following model: $N$ different particles whose mass distribution satisfies equation (5), randomly located on a circle of length $L$, and $L / N=1$. The granular spheres perform a Brownian motion on the one-dimensional ring and interact by means of inelastic collisions.

Between collisions, the movement of each particle obeys the amendatory Langevin equation:

$$
\begin{aligned}
\frac{\mathrm{d} v_{i}}{\mathrm{~d} t} & =-\frac{v_{i}}{\tau}+\sqrt{\frac{2 T_{F}}{m_{i} \tau}} f_{i}(t), \\
\frac{\mathrm{d} x_{i}}{\mathrm{~d} t} & =v_{i}(t),
\end{aligned}
$$

where $1 \leqslant i \leqslant N, \tau$ and $T_{F}$ are the relaxation time due to viscous effects and the thermal bath temperature, respectively, $m_{i}$ is the mass of particles of the $i$ th component, $f_{i}(t)$ is a standard white noise $\left\langle f_{i}(t)\right\rangle=0$, and $\left\langle f_{i}(t) f_{j}\left(t^{\prime}\right)\right\rangle=\delta_{i j} \delta\left(t-t^{\prime}\right)$. $T_{F}$ provides kinetic energy to the system. And due to the random force $f_{i}(t)$, the probability of every particle obtaining energy will be equipotent. After a long time, the system will reach a stationary state as the result of the balance between the dissipation of the kinetic energy due to the collisions between the particles and the energy injection due to the random force.

When collisions are considered, the particles mutually collide according to the following rules: (a) only binary collisions are considered, (b) each collision is instantaneous and (c) the post-collisional velocities are related to the pre-collisional ones by

$$
\begin{aligned}
& v_{i}^{\prime}=\frac{\left(m_{i}-e m_{j}\right) v_{i}+(1+e) m_{j} v_{j}}{m_{i}+m_{j}}, \\
& v_{j}^{\prime}=\frac{(1+e) m_{i} v_{i}+\left(m_{j}-e m_{i}\right) v_{j}}{m_{i}+m_{j}},
\end{aligned}
$$

where $e$ is the restitution coefficient. In this way, the momentum is conserved in the collisions, while the kinetic energy of the centre of the mass is rescaled by $e^{2}$,

$$
\left(v_{i}^{\prime}-v_{j}^{\prime}\right)^{2}=e^{2}\left(v_{i}-v_{j}\right)^{2} .
$$

The elastic case is for $e=1$, while for $e=0$, the colliding particles have no relative motion after the collision. It should be noted that, in one dimension, for the 'hard' nature of collisions, the particles never deform (this effect is taken into account in the restitution coefficient picture), and the length $L / N$ of spacing between particles is much more important than the size of particles.

In the absence of collisions, each particle would perform a Brownian motion, for $t \gg \tau$, a stationary state with a Gaussian velocity distribution:

$$
f\left(v_{i 0}\right) \sim \exp \left(-\frac{m_{i} v_{i 0}^{2}}{2 T_{F}}\right) .
$$

When collisions are considered, another characteristic time emerges, that is the average collision time $\tau_{c}$ between two successive encounters. As a function of average density and 
typical velocity, $\tau_{c}$ can be expressed by

$$
\tau_{c} \sim \frac{L}{2 N \sqrt{\left\langle v^{2}\right\rangle}}
$$

It is usually assumed that $\left\langle v^{2}\right\rangle$ reaches a stationary value with statistical fluctuations (of order $\sim 1 / N)$.

After a long transient, for each given choice of $e$ and $\tau$, the system reaches a stationary state with certain properties. The presence of two time scales, namely $\tau$ and the mean collision time $\tau_{c}$, leads to different dynamical regimes.

(a) When $\tau \ll \tau_{c}$ the effect of collisions is rapidly overwhelmed by the Brownian motion and the system behaves as a collection of weakly interacting random walkers. The particles reach a simple statistical equilibrium and their velocity distribution is that of an ideal gas with a temperature $T_{g}$ being not far from the temperature $T_{F}$ of thermal bath.

(b) In the opposite limit, $\tau \gg \tau_{c}$, the driving mechanism towards the macroscopically stationary state is dominated by the collision process itself. In this regime a statistically stationary state is still observed, and two interesting phenomena emerge: (a) a strongly inhomogeneous spatial distribution, namely clusterization and (b) a deviation of velocity distribution from Gaussian behaviour. These phenomena are more and more pronounced with decreasing values of the fractal dimension $D$.

\section{Simulation and analysis}

\subsection{Simulation}

In all simulations performed, we use $N=9000, \frac{L}{N}=1, m_{\max }=20, n_{0}=1, T_{F}=1$ and $\tau=100 \tau_{c}$. In our simulations, $\frac{M_{\max }}{M_{\min }}>10^{2}\left(M_{\max }\right.$ is the maximal mass of the particles and $M_{\min }$ is the minimal mass of the particles); this inequation satisfies the fractal criterion [29]. It proves that our model has fractal characteristic and our simulations are valid.

First, we randomly give the initial velocity to each particle. In the limit $\tau=100 \tau_{c}$, the collisions dominate the dynamics. Then we perform the simulations by using the Monte Carlo method. The simulations have been performed using a fixed step $\Delta t$ integration of equations (6) and (7) and an event driven check of collisions during every time step, $\Delta t=0.01 \tau_{c}$. After a long time $t=5 \times 10^{6} \Delta t$, the system reaches a stationary state.

Figure 1 shows the distribution of velocities, obtained by sampling the velocities of all particles when the system reaches a stationary state. To testify that the system assuredly reaches a stationary state after the time $t=5 \times 10^{6} \Delta t$, we let the evolution time be $t=4 \times 10^{6} \Delta t$, $t=5 \times 10^{6} \Delta t$ and $t=6 \times 10^{6} \Delta t$, respectively. The figures show that after the system evolves during these three different times, respectively, the three distributions of velocities are almost the same and the lines of the distributions fit well. So we are sure that the system reaches a stationary state after $t=5 \times 10^{6} \Delta t$.

\subsection{Definition of granular temperature}

Marconi and Puglisi [24] studied a Maxwell model of inelastic two-component granular mixture $(\alpha=1,2)$, when the system reaches a stationary state; it appears at two different granular temperatures, one for each species:

$$
T_{\alpha}=\frac{1}{2} m_{\alpha}\left\langle v_{\alpha}^{2}\right\rangle,
$$

where $\alpha=1,2$, is the average performed over the white noise $f_{i}(t)$. Note that these two partial granular temperatures are different and here the granular temperature just means the 

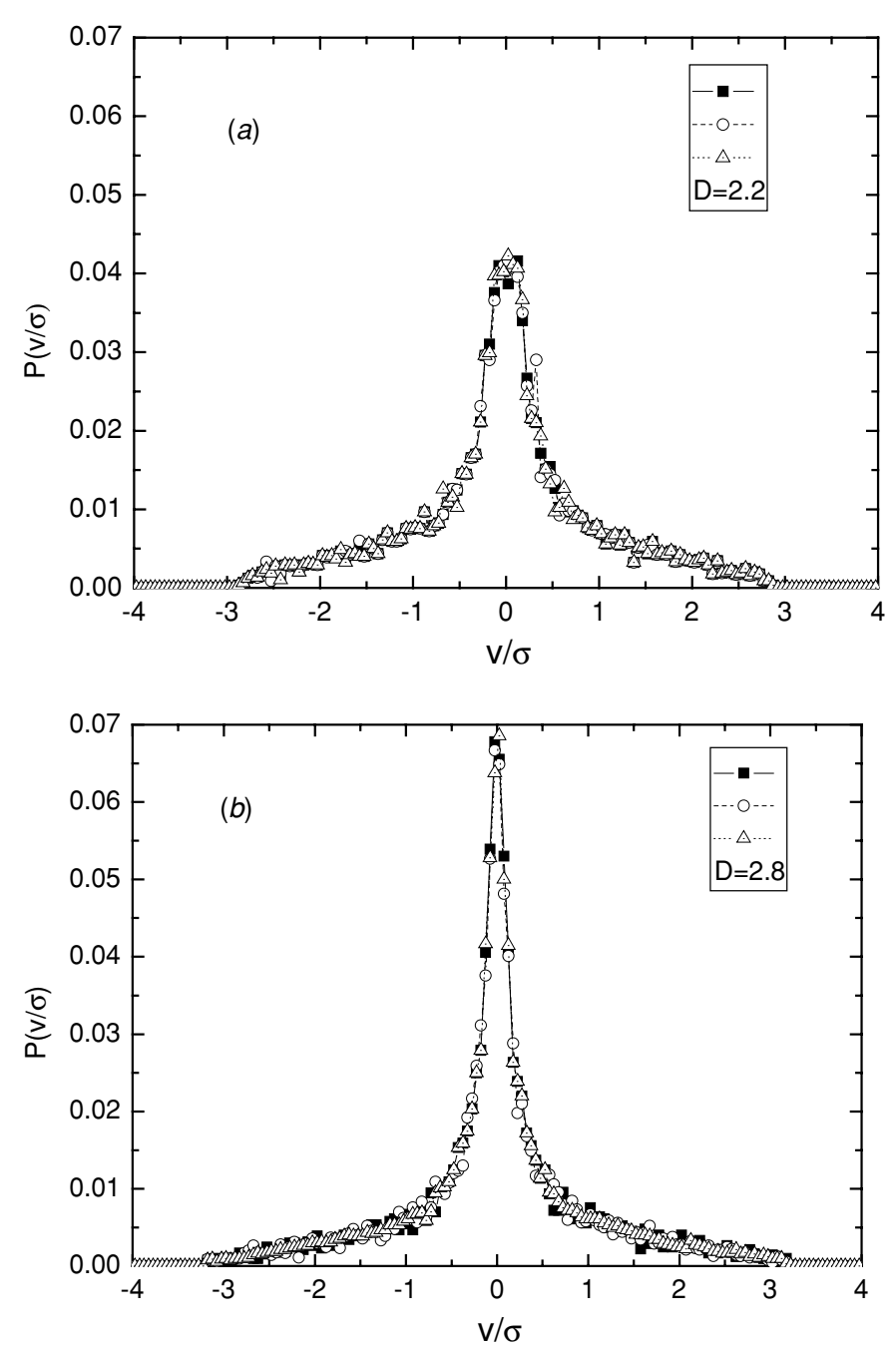

Figure 1. The rescaled velocity distribution $P(v / \sigma)$ versus $v / \sigma$. The fractal dimension is different: (a) $D=2.2$ and (b) $D=2.8$. In both the figures, $\tau=100 \tau_{c}, e=0.6$, the squares represent the evolution time $t=4 \times 10^{6} \Delta t$, circles represent $t=6 \times 10^{6} \Delta t$, triangles represent $t=5 \times 10^{6} \Delta t$.

average kinetic energy per particle of the same component. It is no longer a common feature of all granular gases in mutual equilibrium, i.e., the quantity that has the role of determining if two systems are in equilibrium with respect to each other. In other words, one of the most useful properties of the temperature, i.e., the independence from the thermal substance, is lost when one deals with granular materials. The global temperature of the assembly can be defined by

$$
T_{g}=p T_{1}+(1-p) T_{2}=\left(N_{1} T_{1}+N_{2} T_{2}\right) /\left(N_{1}+N_{2}\right)
$$

where $p=N_{1} /\left(N_{1}+N_{2}\right)$. It is obvious that the global granular temperature is the statistical average value of the partial granular temperatures of the two components. Since the energy dissipation and the energy supply mechanisms compete, the system under the influence of a stochastic white noise driving achieves asymptotically a statistical steady state. Actually the global granular temperature indicates that under the influence of the balance between the 


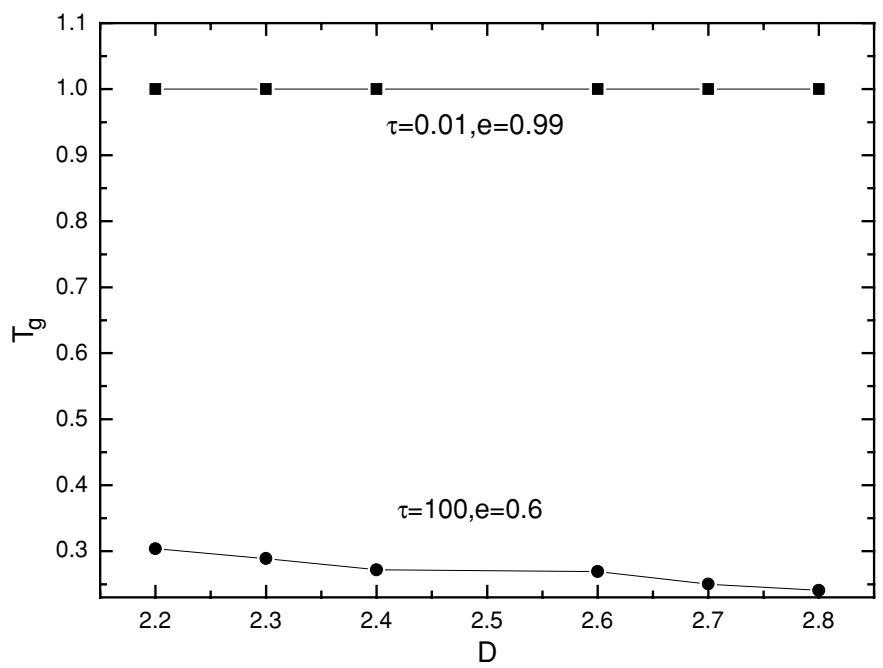

Figure 2. The global granular temperature against the fractal dimension. The top curve represents quasiequilibrium ( $\tau=0.01 \tau_{c}, e=0.99$ ) and the bottom curve means nonequilibrium $\left(\tau=100 \tau_{c}, e=0.6\right)$

energy dissipation and the energy injection due to the external thermal bath, the assembly achieves asymptotically a statistical steady state.

In the spirit of the definition of the partial and global granular temperatures of twocomponent granular mixture, we can define the partial temperatures of the $n$-component mixture as

$$
T_{i}=\frac{1}{2} m_{i}\left\langle v_{i}^{2}\right\rangle,
$$

where $N_{i}$ is the number of particles of the $i$ th component, and the mass of particles of the $i$ th component is $m_{i}$. Here the granular temperature represents the mean kinetic energy per particle of the $i$ th component. The global granular temperature of the $n$-component mixture is

$$
T_{g}=p_{1} T_{1}+p_{2} T_{2}+\cdots+p_{n} T_{n}=\frac{N_{1} T_{1}+N_{2} T_{2}+\cdots+N_{n} T_{n}}{N_{1}+N_{2}+\cdots+N_{n}},
$$

where $p_{i}=N_{i} / N(i=1,2, \ldots, n), p_{1}+p_{2}+\cdots+p_{n}=1, N_{i}$ is the number of particles of the $i$ th component. The total number of the particles of the granular mixture is $N$, and $N=N_{1}+N_{2}+\cdots+N_{n}$. Here the global granular temperature is the statistic average value of the partial granular temperatures of the $n$ components and also indicates that the assembly achieves asymptotically a statistical steady state, as the energy dissipation is balanced by the energy injection. It is convenient to define the global and partial granular temperatures of the granular mixture whose mass distribution is continuous by means of the definition of the global and partial granular temperatures of the $n$-component mixture, which is testified in our another paper 'Granular temperatures of driven inelastic multi-component mixture'.

\subsection{Global granular temperature and dissipated energy per particle}

In figure 2, we report the global granular temperature $T_{g}$ versus the fractal dimension $D$ for different $e$ and $\tau$. The system behaves as a collection of weakly interacting random walkers when the restitution coefficient $e=0.99, \tau=0.01 \tau_{c}$. So the global granular temperature $T_{g}$ does not change with different fractal dimensions $D$, and $T_{g}=1$. Actually the partial granular 


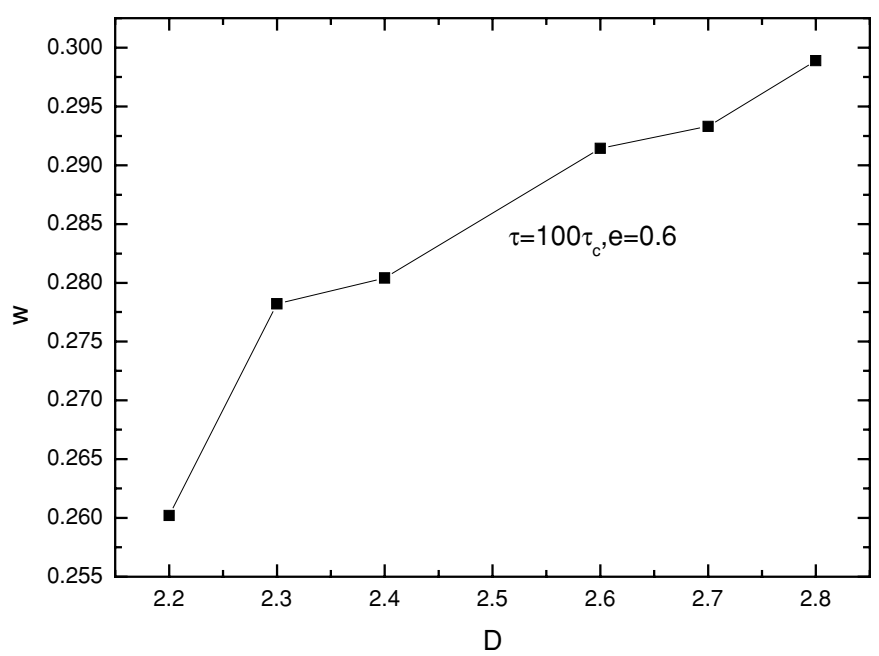

Figure 3. Dissipated energy per particle $w$ versus fractal dimension $D$ for $e=0.6, \tau=100 \tau_{c}$, $N=9000$.

temperature per component is also the same and equal to 1 . When $e=0.6, \tau=100 \tau_{c}$, the collisions dominate the dynamics and strongly compete against the driving mechanism. Therefore, the global granular temperature of the system decreases as the fractal dimension $D$ increases. The increasing value of $D$ indicates that the inhomogeneity in the size distribution of the system increases.

In figure 3 , we show the dissipated energy per particle versus the fractal dimension $D$. Because the driving mechanism is dominated by the inelastic collision process, the dissipated energy per particle $w$ increases as the fractal dimension $D$ increases.

\subsection{Probability distribution of velocity in one-dimensional case}

Figure 4 shows the distribution of velocities obtained by sampling the velocities of all particles in two different regimes (a quasiequilibrium case with $\tau=0.01 \tau_{c}, e=0.99$ and an out-ofequilibrium case with $\tau=100 \tau_{c}, e=0.6$ ) when the system has reached a statistic stationary state. In the quasiequilibrium regime the velocity distribution which is limned in squares, is very well fitted by Gaussian. When $\tau=100 \tau_{c}, e=0.6$ the velocity distribution which is described by rotundities, ceases to be Gaussian. The deviation becomes more and more pronounced as the fractal dimension $D$ increases.

\subsection{Instantaneous density of particles in one-dimensional case}

In figure 5 we report different density profiles in the two regimes (homogeneous and clusterized). From the figures we can see when the system attains a statistically stationary state, the instantaneous density of particles becomes strongly inhomogeneous. The spatial clusterization becomes more and more pronounced with increasing values of the fractal dimension $D$.

The clusterization may quantitatively be characterized by means of an entropy defined as

$$
h_{M}=-\sum_{j=1}^{M} \frac{m_{j}}{N} \ln \frac{m_{j}}{N}
$$



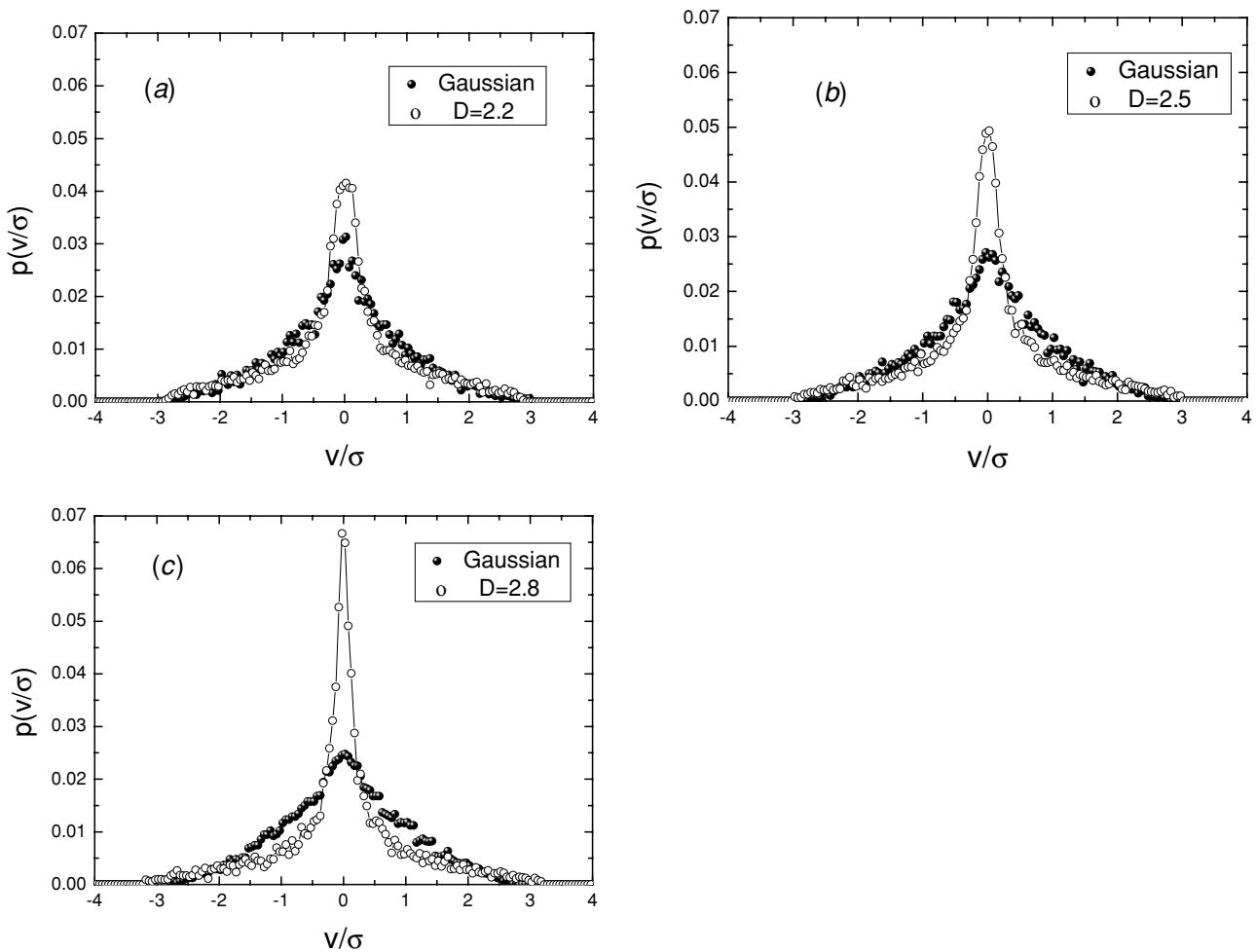

Figure 4. The rescaled velocity distribution $P(v / \sigma)$ versus $v / \sigma$ for different values of $D$ : (a) $D=2.2$, (b) $D=2.5$ and (c) $D=2.8$ with $\tau=100 \tau_{c}, e=0.6, N=9000$.

where the ring of length $L$ is divided into $M$ equal boxes (i.e., segments) and $m_{j}$ is the number of particles in the $j$ th box. The entropy $h_{M}$ attains its maximum value $h_{M}=\ln m$ when $m_{j}=N / M$ for every box $j . h_{M}$ decreases as the density distribution becomes more and more clusterized.

In figure 6 many measurements of $H_{M} / H_{M}^{*}$ are presented, where $H_{M}=\exp \left(\left\langle h_{M}\right\rangle\right), H_{M}^{*}=$ $\exp \left(\left\langle h_{M}^{*}\right\rangle\right), h_{M}^{*}$ is the effective entropy for homogeneous regime $\left(\tau=0.01 \tau_{c}, e=0.99\right)$ and \langle\rangle is the time average. The quantity $H_{M} / H_{M}^{*}$ basically gives an indication of the fraction of non-empty boxes in a typical snapshot.

The figure shows that $H_{M} / H_{M}^{*}$ decreases as the fractal dimension $D$ increases with respect to the same restitution coefficient $e$. Namely, the system becomes more and more clusterized. When $e=1$, all the curves reach one point and $H_{M} / H_{M}^{*}$ attains its maximum value. The spatial density is homogeneous and there is no energy dissipation as the collisions between particles are elastic. The energy dissipation induces clusterization.

\subsection{Analysis}

In all our simulations, the collisions between the particles are inelastic. From equations (8) and (9), the energy dissipation due to the inelastic collisions during one collision is

$\Delta E=\frac{\left(1-e^{2}\right)\left(v_{i}+v_{j}\right)^{2}}{2} \cdot \frac{m_{i} m_{j}}{m_{i}+m_{j}}=\frac{\left(1-e^{2}\right)\left(v_{i}+v_{j}\right)^{2}}{2} \cdot \frac{m_{i}\left(m_{i}+\Delta m_{i j}\right)}{2 m_{i}+\Delta m_{i j}}$, 

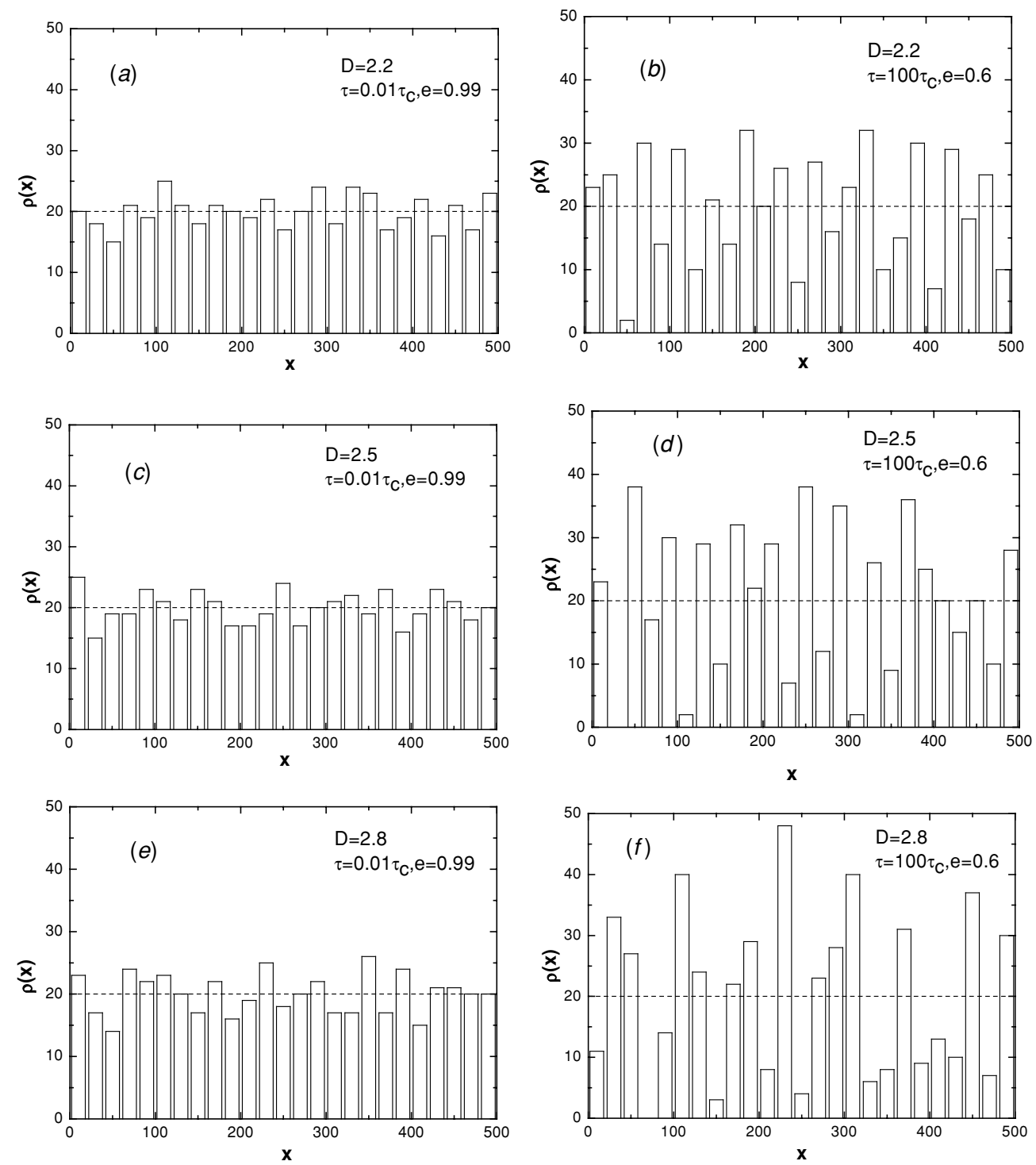

Figure 5. Instantaneous density profiles $\rho(x)$ in two regimes: $(a),(c),(e)$ quasiequilibrium regime ( $\tau=0.01 \tau_{c}, e=0.99$ ) with $D=2.2, D=2.5, D=2.8$, respectively, and $(b),(d),(f)$ nonequilibrium regime with clusters $\left(\tau=100 \tau_{c}, e=0.6\right)$ with $D=2.2, D=2.5, D=2.8$, respectively. In all histograms $N=500$ and the dashed horizontal lines represent the average density, equal to 20 particles per bin.

where $\Delta m_{i j}=m_{i}-m_{j}$. Obviously, when the restitution coefficient $e$ does not change, the difference of the mass between the two colliding particles is greater, the dissipation of energy $\Delta E$ is higher. As the increasing value of $D$ represents more number of finer particles in the system, the inhomogeneity of size distribution is more prominent, which makes the difference of the mass between any two colliding particles greater. So the higher the value of $D$, the more dissipated the energy of the system. Therefore, with the increasing value of $D$, the 


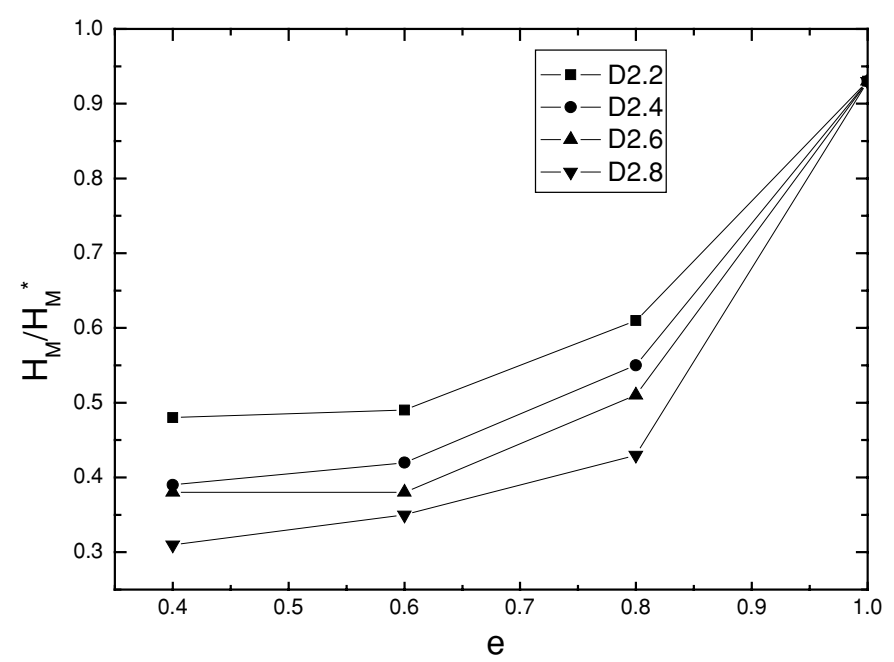

Figure 6. $H_{M} / H_{M}^{*}$ versus $e$ for different $D$ : from top to bottom $D$ is $2.2,2.4,2.6$, 2.8, with $N=500, M=500, e=0.6, \tau=100 \tau_{c}$.

global granular temperature decreases, the average dissipated energy per particle increases and the velocity probability distribution deviates more obviously from the Gaussian one. The density distribution becomes more and more clusterized as the energy dissipation is more pronounced.

\section{Conclusion}

In this paper, we represent a fractal model of a non-uniform driven inelastic granular system and an amendatory Langevin equation which each particle of the system obeys. By means of fractal theory, we have studied the nonequilibrium properties of the system. Note that the system is not at equilibrium, so granular temperature is not a temperature in a proper thermodynamic sense. We define the partial and global granular temperatures of the mixture. The partial granular temperature is the average kinetic energy per particle of the same component and the global one is the statistic average value of partial granular temperatures of $n$ components. One of the most useful properties of the temperature, i.e., the independence from the thermal substance is lost.

We represent that the fractal dimension $D$ is a measurement of the inhomogeneity of the size distribution. So the higher the $D$, the more inhomogeneous the size distribution.

Our simulation results show when $e=0.6, \tau=100 \tau_{c}$, the global granular temperature reduces and average dissipated energy per particle increases with the augment of $D$. However, in the quasiequilibrium regime $\left(\tau=0.01 \tau_{c}, e=0.99\right)$ the global granular temperature is independent of $D$ and the partial granular temperature per component is equal and up to the global one.

When $\tau \gg \tau_{c}$, non-Gaussian velocity distribution and clusterization arise. The deviation and clusterization become more and more pronounced as the fractal dimension $D$ increases. The clusterization is quantitatively characterized by means of an entropy $h_{M}$. The dynamics properties of the non-uniform granular system are prominently influenced by the size distribution of the system. 


\section{Acknowledgments}

This work was supported by the National Natural Science Foundation of China through grant no 50272022 and Sunshine Foundation of Wuhan city through grant no 20045006071-40.

\section{References}

[1] Campbell C S 1990 Annu. Rev. Fluid Mech. 2257

[2] Jaeger H M, Nagel S R and Behringer R P 1996 Phys. Today 4932

Jaeger H M, Nagel S R and Behringer R P 1996 Rev. Mod. Phys. 681259

[3] Kadanoff L P 1999 Rev. Mod. Phys. 71435

[4] Duran J 2000 Sands, Powders and Grains. An Introduction to the Physics of Granular Materials (New York: Springer)

[5] Pöschel T and Luding S (ed) 2001 Granular Gases (Lecture Notes in Physics vol 564) (Berlin: Springer)

[6] McNamara S and Young W R 1992 Phys. Fluids A 4496

McNamara S and Young W R 1994 Phys. Rev. E 50 R28

[7] Rouyer F and Menon N 2000 Phys. Rev. Lett. 853676

[8] Olafsen J S and Urbach J S 1998 Phys. Rev. Lett. 814369

Olafsen J S and Urbach J S 1999 Phys. Rev. E 60 R2468

[9] Kudrolli A, Wolpert N and Gollub J P 1997 Phys. Rev. Lett. 781383

[10] Losert W, Cooper D G W and Gollub J P 1999 Phys. Rev. E 595855

[11] Blair D L and Kudrolli A 2001 Phys. Rev. E 64050301

[12] Goldhirsch I and Zanetti G 1993 Phys. Rev. Lett. 701619

[13] van Noije T P C and Ernst M H 1998 Granular Matter 158

[14] Carrillo J A, Cercignani C and Gamba I M 2000 Phys. Rev. E 627700

[15] Moon S J, Shattuck M D and Swift J B 2001 Phys. Rev. E 64031303

[16] Cafiero R, Luding S and Herrmann H J 2000 Phys. Rev. Lett. 846014

[17] Soto R, Piasecki J and Mareschal M 2001 Phys. Rev. E 64031306

[18] Du Y, Li H and Kadanoff L P 1995 Phys. Rev. Lett. 741268

[19] Grossman E L, Zhou T and Ben-Naim E 1997 Phys. Rev. E 554200

[20] Esipov S E and Pöshcel T 1997 J. Stat. Phys. 861385

[21] Puglisi A et al 1998 Phys. Rev. Lett. 813848

[22] Puglisi A, Loreto V, Marini Bettolo Marconi U and Vulpiani A 1999 Phys. Rev. E 595582

[23] Williams D R M and MacKintosh F C 1996 Phys. Rev. E 54 R9

[24] Marini Bettolo Marconi U and Puglisi A 2002 Phys. Rev. E 65051305 Marini Bettolo Marconi U and Puglisi A 2002 Phys. Rev. E 66011301

[25] Pagnani R, Marini Bettolo Marconi U and Puglisi A 2002 Phys. Rev. E 66051304

[26] Zhang D M, Zhang Z and Yu B M 1999 Commun. Theor. Phys. 31373

[27] Zhang D M, Lei Y J and Yu B M 2002 Commun. Theor. Phys. 37231

[28] Zhang D M, Lei Y J, Pan G J and Yu B M 2003 Chin. Phys. Lett. 202221

[29] Yu B M and Li J H 2001 Fractals 9 365-72 Yu B M et al 2002 Fractals 10135

[30] Zhang L M 2002 Fractal characteristics of granularity distributions of aqueous bentonite and betonite-polymer muds Colloids Surf. A 202 1-7 